\title{
From crude industrial waste glycerol to biopropene via Ru-mediated hydrodeoxygenation in ionic liquids
}

Received 00th January 20xx,

\author{
Kwinten Janssens, Maxime Stalpaert, Mickaël Henrion and Dirk E. De Vos*
}

Accepted 00th January 20xx

DOI: $10.1039 / \times 0 \times x 00000 x$

The catalytic conversion of crude glycerol to biopropene was investigated. A bifunctional Ru-ionic liquid system showed a high tolerance for common crude glycerol impurities like water, salts and methanol. After optimizing both dehydration and olefin selectivity, a $82 \%$ biopropene yield ( $94 \%$ selectivity) was obtained directly from industrial waste glycerol.

The global increase in biofuel consumption for transportation leads to an accumulation of waste glycerol (approximately $10 \mathrm{wt} \%$ in plant oils) with an expected annual production of 41.9 billion litres of crude glycerol in 2020. ${ }^{1}$ This crude glycerol generally contains impurities, of which water, $\mathrm{NaCl}$ and $\mathrm{MeOH}$ are considered the most important ones. ${ }^{2-4}$ Purified glycerol has applications as an added-value chemical in the pharmaceutical, food and cosmetic industry. ${ }^{5-8}$ Unfortunately, these applications require an energy intensive purification, consisting of filtration, chemical additions, and fractional vacuum distillation to obtain high grade glycerol with adequate product performance and safety. For lower grade glycerol, treatment with a series of ion exchange resins might be sufficient. ${ }^{8}$ For the conversion of glycerol to high-value end products, the required pre-purification steps limit the value of glycerol as a platform molecule. ${ }^{9,10}$ Conversely, if glycerol is catalytically converted to lower added value polymer precursors, e.g. olefins, the economically preferred solution is to start directly from the waste glycerol without the expensive additional purifications. ${ }^{11}$ This requires a catalytic system that is able to tolerate the typical impurities. Over the last decade, several groups have described the conversion of glycerol to propene, the best results of which are summarized in the ESI (Table S1). ${ }^{12-19}$ While high conversion of glycerol and selectivity to propene have been reached, only few reports describe (simulated) waste glycerol blends. Bimetallic heterogeneous catalysts have been proposed for the direct hydrodeoxygenation (HDO) of glycerol to olefins (GTO) using continuous fixed-bed gas-phase reactors. ${ }^{12-16}$ These systems generally rely on a tandem process in which a selective metal mediates hydrogenolysis of glycerol to 1-propanol, followed by acid catalysed dehydration. ${ }^{15}$ However, these reactions are not reported to tolerate any remaining salt

cMACS, KU Leuven, Celestijnenlaan 200F, 3001 Leuven, Belgium.

E-mail: dirk.devos@kuleuven.be

†Electronic Supplementary Information (ESI) available. Literature summary,

Experimental procedures, Crude glycerol analysis, Catalyst recycling experiments. See DOI: $10.1039 / \mathrm{x} 0 \times x 00000 \mathrm{x}$ impurities. Salts obviously are highly problematic in evaporation of the feed, easily leading to reactor obstruction with solids, or to salt deposition on the catalyst. Moreover, significant dilution is needed in order to vaporize glycerol into the gaseous phase, the latter leading to considerable energy consumption, in addition to the required desalination process. Foregoing the salt issues, only $\mathrm{Wu}$ et al. were able to design a heterogeneous catalyst that could handle simulated crude glycerol with a rather realistic glycerol-water-methanol ratio. ${ }^{15,16}$ Unfortunately, the cracking temperature had to be increased from $250^{\circ} \mathrm{C}$ to $450^{\circ} \mathrm{C}$ to allow the system to work with $\pm 5 \mathrm{wt} \%$ methanol, leading to increased coking. Alternatively, an entirely different system was reported applying homogeneous acids (e.g. hydrogen iodide or triflic acid) in combination with a transition metal catalyst. ${ }^{17-19}$ These acids had a significant impact on the stainless steel reactors, leading to ill-defined catalyst species and possibly significant metal leaching into the reaction medium. More recently, our group discovered a novel approach to perform the HDO of biobased polyols using a combination of a homogeneous hydrogenation catalyst and a dehydrating solvent of the ionic liquid type (IL; tetrabutylphosphonium bromide, $\left.\mathrm{Bu}_{4} \mathrm{PBr}\right){ }^{20}$ Our previous work focused on the conversion of higher polyols (i.e. erythritol, xylitol and sorbitol). Here, we further explore this catalytic system in order to evaluate its relevance and practical applicability for industrial crude glycerol, as obtained from biodiesel production.

Our original $\mathrm{Ru} / \mathrm{Bu}_{4} \mathrm{PBr}$ system contained $\mathrm{RuBr}_{3}$ as the catalyst precursor, formalin as an additive, together with $\mathrm{HBr}$ doped $\mathrm{Bu}_{4} \mathrm{PBr}$ as a dehydration catalyst. Extensive experiments with $\mathrm{C}_{4}-\mathrm{C}_{6}$ polyols showed that temperature plays a crucial role in balancing the dehydration with the hydrogenating activity of the in situ formed Ru species, converting carbonyl compounds to alcohols. ${ }^{20}$ In the case of erythritol, the dehydration by the IL proved to be more rate limiting at low temperature, while at elevated temperature the hydrogenation of butanone was identified as the kinetically slowest step. ${ }^{20}$ High temperatures also increase to risk of overreduction of the desired olefin product to the alkane. 

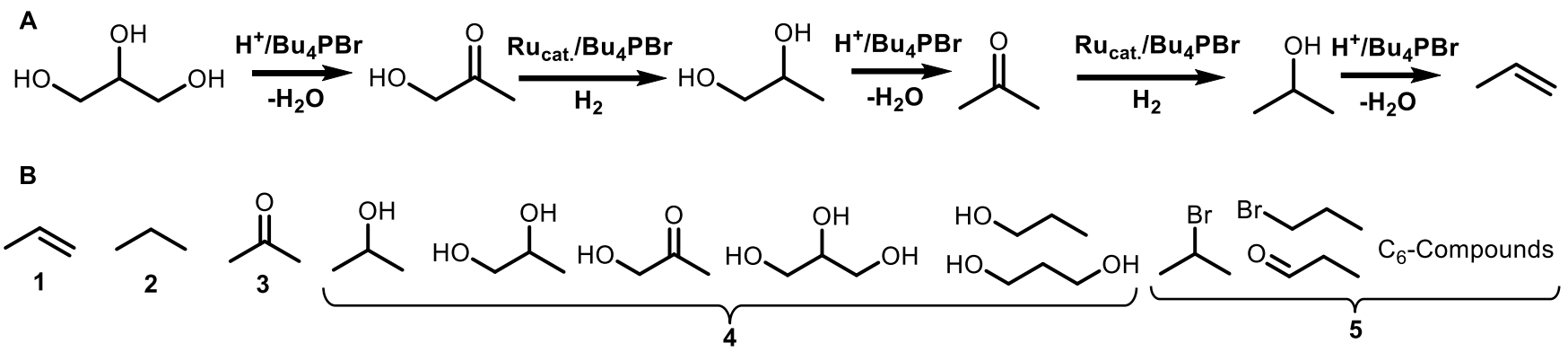
observed (side) products and reaction intermediates are presented (1-5). Details on the product analysis is provided in the ESI.

When applying the existing system to analytically pure glycerol, a significant propene yield of $57 \%$ was obtained after $1 \mathrm{~h}$ at $210^{\circ} \mathrm{C} .{ }^{20}$ The proposed main reaction pathway for the complete HDO of glycerol is illustrated in Scheme 1, based on previous observations in the HDO of sugar alcohols and glycerol. ${ }^{12,15,20}$ However, the selectivity was not maintained when the reaction time was increased to convert the remaining hydroxy compounds in full; in an attempt to boost propene yields, an olefin selectivity drop was observed with a $10 \%$ increase in propane yield after $2 \mathrm{~h}$ (Scheme 1 shows all observed products).

In our approach to valorise industrial waste glycerol, the first test was to verify the water tolerance of this existing, nonoptimized Ru system. Previous reports on dehydration reactions in $\mathrm{HBr} / \mathrm{Bu}_{4} \mathrm{PBr}$ showed activity is preserved over multiple cycles, despite the significant water build-up. ${ }^{20,21}$ Glycerol-water simulated blends were evaluated, ranging from pure glycerol to $50 \mathrm{wt} \%$ glycerol in water. The latter feed contains over 5 equivalents of water, in addition to the 3 equivalents potentially formed by the dehydration. Since avoiding overreduction is critical at higher temperatures, the

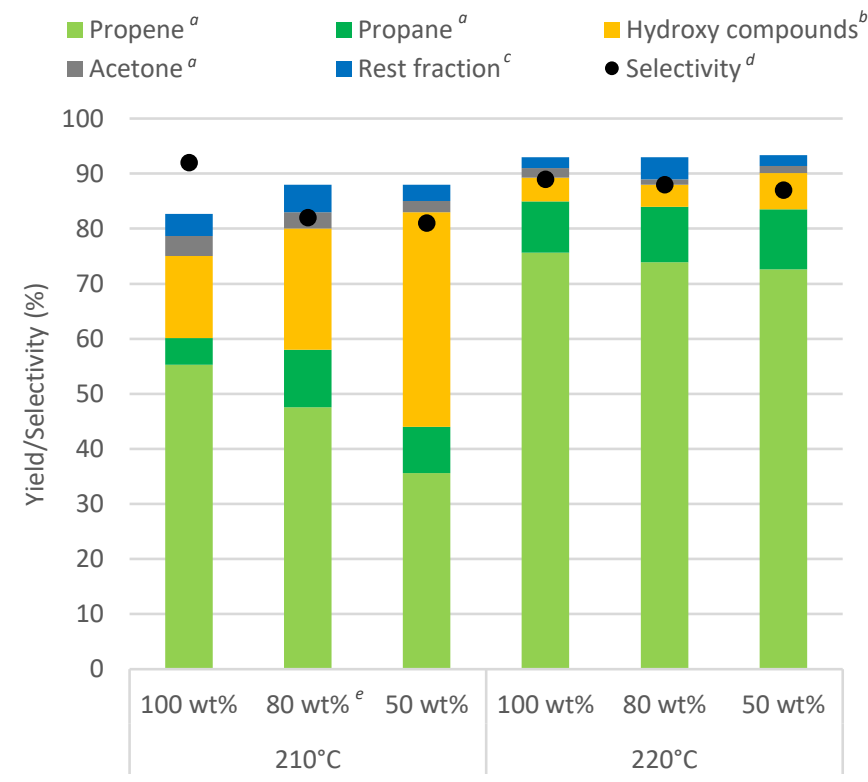

Fig. 1 HDO of water-diluted glycerol at various temperatures. ${ }^{a} \mathrm{GC}$ yield ${ }^{b}$ Combined GC yield of hydroxy compounds (4) after derivatisation. ${ }^{c}$ Combined GC yield of bromopropanes, propanal and $\mathrm{C}_{6}$ compounds (5). ${ }^{d}$ Selectivity for propene (1) in the (propene + propane) fraction. e Glycerol dilution in water (wt\%); 80wt\% represents a blend of $80 \mathrm{mg}$ pure glycerol and $20 \mathrm{mg}$ deionized water. (Detailed procedure is presented in the ESI and Table 1, entry 1 ). selectivity for propene ( $\mathbf{1}$ vs $\mathbf{2}$ ) is shown separately in Fig. 1 . At $210^{\circ} \mathrm{C}$, significant decreases in both dehydration rate and propene versus propane selectivity were observed for high water loadings. Fortunately, this was easily circumvented by working at a slightly elevated temperature of $220^{\circ} \mathrm{C}$, resulting in a significant increase in overall propene yield compared to the best literature result ( $76 \%$ vs $57 \%$, after $1 \mathrm{~h})$. High conversions and selectivities were retained, especially at high water content. Thus, a slight increase of reaction temperature readily allows to avoid pre-distillation of the glycerol feed to remove water. ${ }^{9,10}$

Moving closer to crude glycerol, we tested the ability of the catalytic system to tolerate other typical glycerol impurities (Table 1, entries 2-9)..$^{2-4}$ The addition of $5 \mathrm{wt} \% \mathrm{MeOH}$ (entry 2) resulted in a similar product distribution and selectivity as obtained with diluted pure glycerol ( $80 \mathrm{wt} \%$ in water). However, the addition of $\mathrm{NaCl}$ led to a significant decrease in both selectivity and yield at higher loadings (entries 3-5). $\mathrm{NaCl}$ can be expected to be present in up to $5 \mathrm{wt} \%$ after the triglyceride saponification and acidification steps. The influence of the anion and cation was investigated separately (entry 6-8). An effect of $\mathrm{Na}^{+}$can be excluded, since the addition of $\mathrm{NaBr}$ has no significant impact compared to the reference. By contrast, $\mathrm{KCl}$ leads to a decrease in the yield of fully deoxygenated $\mathrm{C} 3$ compounds $(\mathbf{1 + 2})$, and an increase of $\mathrm{OH}$-functionalized intermediates like isopropanol and 1,2-propanediol, indicating a decreased dehydration rate upon addition of $\mathrm{Cl}^{-}$anions. The slower dehydration of hydroxylated intermediates also produces a small adverse effect on the propene selectivity as previously observed in Fig. 1 . The selectivity and yield remain higher with the addition of $\mathrm{KBr}$ as an additive, again excluding an effect of a cation like $\mathrm{K}^{+}$. The combination of $5 \mathrm{wt} \% \mathrm{MeOH}$ and $5 \mathrm{wt} \% \mathrm{NaCl}$ in diluted glycerol is an accurate, albeit extreme simulation of industrial crude glycerol containing the most abundant traces. This simulated blend was compared with real ex-biodiesel crude waste glycerol obtained directly from the natural chemistry industry (entries 9,10 ). Both simulated and real crude glycerol illustrate the potential applicability of the new catalytic system, with approximately $80 \%$ yield of fully deoxygenated $\mathrm{C} 3(\mathbf{1 + 2})$ and, within this fraction, $75 \%$ propene selectivity, amounting to $62 \%$ propene yield. While promising, these results still show room for improvement, especially in the olefin selectivity. 
Table 1: Hydrodeoxygenation of (simulated) waste glycerol ${ }^{a}$

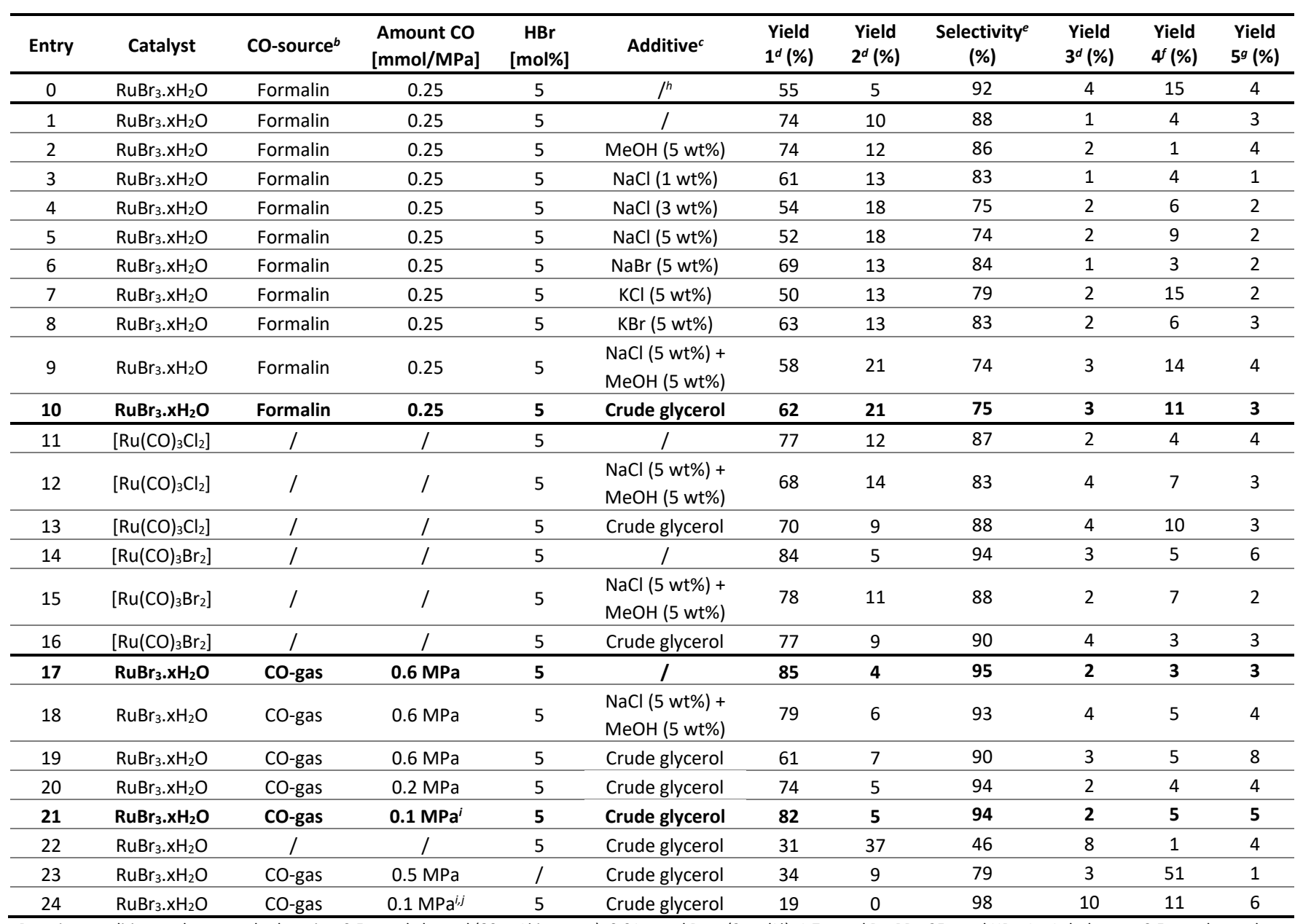

${ }^{a}$ Reaction conditions unless stated otherwise: $0.5 \mathrm{mmol}$ glycerol ( $80 \mathrm{wt} \%$ in water), $0.01 \mathrm{mmol} \mathrm{Ru}$ cat $(2 \mathrm{~mol} \%), 1.7 \mathrm{mmol} \mathrm{Bu} \mathrm{PBr}_{2} 25 \mu \mathrm{mol} \mathrm{HBr}, 1 \mathrm{~mL}$ dodecane, $0.5 \mathrm{mmol}$ tetradecane (IS), $220^{\circ} \mathrm{C}, 4.0 \mathrm{MPa} \mathrm{H}$, $1 \mathrm{~h}$. ${ }^{b}$ Pretreatment of $\mathrm{RuBr}_{3}$ with $\mathrm{CO}$ source, $30 \mathrm{~min}, 4.0 \mathrm{MPa} \mathrm{H}_{2}, 180^{\circ} \mathrm{C}$. ${ }^{c}$ Added to 80 wt\% glycerol in water. ${ }^{d} \mathrm{GC}$ yield ${ }^{e}$ Ratio of propene vs (propene + propane) determined by FT-IR gas analysis. ${ }^{f}$ Combined GC yield of hydroxy compounds (4) after BSTFA derivatisation. ${ }^{g}$ Combined GC yield of bromopropanes, propanal and $\mathrm{C}_{6}$ compounds (5). ${ }^{h}$ Benchmark reaction ${ }^{20}$ on pure glycerol at $210^{\circ} \mathrm{C} .{ }^{i}$ Reactor flushed 3 times with pure $\mathrm{CO}$ gas, followed by $\mathrm{H}_{2}$ loading. ${ }^{j} \mathrm{No}$ pretreatment, $\mathrm{CO}$ supplied during reaction.

The crucial role of CO ligands was studied earlier for the HDO of longer polyols. ${ }^{20}$ While we previously used an optimized amount of formalin to in situ form $\mathrm{CO}$ which coordinates on Ru, an alternative option is to use well-defined ruthenium carbonyl catalysts $\left[\mathrm{RuX}_{2}(\mathrm{CO})_{3}\right]_{2}(\mathrm{X}=\mathrm{Br}, \mathrm{Cl})$. In an attempt to improve the propene selectivity, we tested these commercial $(\mathrm{Cl})$ and selfsynthesized $22(\mathrm{Br})$ Ru carbonyl halide catalysts on simulated and real crude glycerol blends (entries 11-16). For both catalysts, results indicate that the olefin selectivity is improved. However, use of $\left[\mathrm{RuCl}_{2}(\mathrm{CO})_{3}\right]_{2}$ also leads to a slight increase of hydroxy intermediates, presumably due to the higher $\mathrm{Cl}$ content and the associated decreased dehydration ability. Remarkably, this is not the case for $\left[\operatorname{RuBr}_{2}(\mathrm{CO})_{3}\right]_{2}$ : increases in propene selectivity and yield are observed compared to the formalin pretreatment (entries 15, 16 vs 9,10 ).

In order to avoid the cumbersome synthesis of $\left[\operatorname{RuBr}_{2}(\mathrm{CO})_{3}\right]_{2}$, we next aimed at achieving similar performance through pretreatment of $\mathrm{RuBr}_{3}$ in a $\mathrm{CO}$ atmosphere. This approach was generally highly beneficial for the propene selectivity in the $(1+2)$ fraction, with values $>90 \%$ being reached in all reactions of entries 17-21. As a starting point, 0.6 MPa of $\mathrm{CO}$ overpressure was tested during pretreatment, showing a promising $85 \%$ net propene yield from purified glycerol (entry 17). Unfortunately, a decrease was observed when using crude glycerol. Since there was no build-up of hydroxylated compounds (4), this decrease does not seem a consequence of slow dehydration. Rather, the deficiencies in the carbon balances $(80-90 \%$, compared to the standard $>90 \%$ ) suggest inhibition of the Ru hydrogenation catalyst by the high $\mathrm{CO}$ content. ${ }^{20}$ As a result, carbonyl compounds (e.g. hydroxyacetone, acetone) are only slowly hydrogenated, but also lost by aldol condensation to $\mathrm{C} 6$ and higher products. Luckily, this could be circumvented using less CO during the pretreatment; use of only atmospheric pressure of CO leads to an optimal olefin selectivity (94\%) and propene net yield (82\%) obtained directly from industrial crude glycerol (entry 21). The necessity of a CO-source and acid co-catalyst was verified (entries 22, 23), illustrating the importance of $\mathrm{CO}$ for the catalyst's selectivity and the extra acid to achieve adequately fast dehydration. 


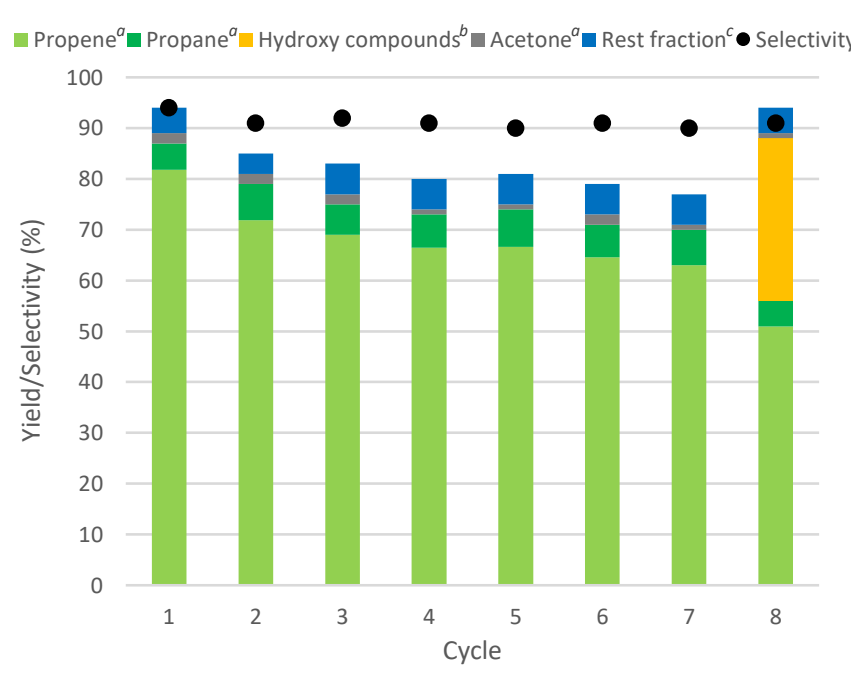

Fig. 2 HDO of crude glycerol in multiple cycles. ${ }^{a} \mathrm{GC}$ yield ${ }^{b}$ The combined GC yield of hydroxy compounds (4) was only determined after the $8^{\text {th }}$ cycle. ${ }^{c}$ Combined GC yield of bromopropanes, propanal and $C_{6}$ compounds (5). ${ }^{d}$ Selectivity for propene (1) in the (propene + propane) fraction. (The recycling details are presented in ESI)

Lastly, the HDO was tested under constant CO atmosphere (entry 24); when the $\mathrm{CO}$ is still present during the reaction, the resulting hydrogenation catalyst is clearly inhibited as shown by the high abundance of carbonyl species and poor mass balance, as a consequence of aldol condensations. This confirms the crucial need for a separate catalyst pretreatment step.

Recycling tests indicated a good recyclability of the catalytic system for up to 8 cycles, maintaining a constant, high selectivity of over $90 \%$ towards the desired olefin (Fig. 2). The accumulation of water and other impurities is expected to be have a negative effect on the catalytic performance. After each cycle a slight decrease in propene yield is observed compared to the fresh catalyst, presumably through $\mathrm{Cl}^{-}$build-up leading to decreased dehydration by the IL. This was confirmed by the relatively large amount of hydroxy compounds present after the $8^{\text {th }}$ cycle. Remarkably, the high selectivity could be retained in full with water removal and CO pretreatment in between each catalytic run. Solid phase FT-IR indicates no significant difference between fresh and recycled catalysts (Fig. S1). Upon exposing fresh and used catalysts to propylene and $\mathrm{H}_{2}$, hardly any propane is formed, proving that the catalyst's selectivity is preserved over multiple cycles.

In conclusion, high propene yields were achieved with a catalytic system combining a $\mathrm{HBr} / \mathrm{Bu}_{4} \mathrm{PBr}$ dehydrating solvent with a very selective Ru hydrogenation catalyst, even when starting directly from industrial waste glycerol. Analysis of the effects of the most common impurities in simulated blends revealed the need for a better catalyst pretreatment to maintain high propene selectivity. Pretreatment of the simple $\mathrm{RuBr}_{3}$ salt under atmospheric CO pressure, and slightly increasing the reaction temperature to $220^{\circ} \mathrm{C}$ allowed to selectively convert ex-biodiesel waste glycerol to $82 \%$ biopropene, with $94 \%$ selectivity in the C3 hydrocarbon fraction.

K.J. and D.D.V. are grateful to Oleon NV 'A Natural Chemistry' for a gift of ex-biodiesel waste glycerol. K.J. and D.D.V. acknowledges the FWO for funding (G0D0518N, G0781118N and GOF2320N), the Flemish government for long-term structural funding through Methusalem, and EoS (Biofact) for financial support.

\section{Conflicts of interest}

There are no conflicts to declare.

\section{Notes and references}

1 C. J. A. Mota, B. Peres Pinto and A. L. de Lima, in Glycerol, Springer International Publishing, Cham, 2017, pp. 11-19.

2 J. C. Thompson and B. B. He, Appl. Eng. Agric., 2006, 22, 261-265.

A. Dobrowolski, P. Mituła, W. Rymowicz and A. M. Mirończuk, Bioresour. Technol., 2016, 207, 237-243. S. Hu, X. Luo, C. Wan and Y. Li, J. Agric. Food Chem., 2012, 60, 5915-5921.

5 D. T. Johnson and K. A. Taconi, Environ. Prog., 2007, 26, 338-348.

M. Pagliaro and M. Rossi, The Future of Glycerol, The Royal Society of Chemistry, 2008.

7 M. Ayoub and A. Z. Abdullah, Renew. Sustain. Energy Rev. 2012, 16, 2671-2686.

8 X. Luo, X. Ge, S. Cui and Y. Li, Bioresour. Technol., 2016, 215, 144-154.

G. M. Lari, G. Pastore, M. Haus, Y. Ding, S.

Papadokonstantakis, C. Mondelli and J. Pérez-Ramírez, Energy Environ. Sci., 2018, 11, 1012-1029.

S. Bagheri, N. M. Julkapli and W. A. Yehye, Renew. Sustain. Energy Rev., 2015, 41, 113-127.

11 Z. Y. Zakaria, N. A. S. Amin and J. Linnekoski, Biomass and Bioenergy, 2013, 55, 370-385.

12 C. J. A. Mota, V. L. C. Gonçalves, J. E. Mellizo, A. M. Rocco, J. C. Fadigas and R. Gambetta, J. Mol. Catal. A Chem., 2016, 422, 158-164.

13 V. Zacharopoulou, E. S. Vasiliadou and A. A. Lemonidou, Green Chem., 2015, 17, 903-912. D. Sun, Y. Yamada and S. Sato, Appl. Catal. B Environ., 2015, 174-175, 13-20.

15 Z. Wu, K. Zhao, S. Ge, Z. Qiao, J. Gao, T. Dou, A. C. K. Yip and M. Zhang, ACS Sustain. Chem. Eng., 2016, 4, 41924207.

Z. Wu, H. Yan, S. Ge, J. Gao, T. Dou, Y. Li, A. C. K. Yip and M. Zhang, Catal. Commun., 2017, 92, 80-85. Deshpande, R. Davis, P. Pandey, V. Kore, N., US Pat., WO20130900, 2013

W. Yang, M. R. Grochowski and A. Sen, ChemSusChem, 2012, 5, 1218-1222.

19 D. Di Mondo, D. Ashok, F. Waldie, N. Schrier, M. Morrison and M. Schlaf, ACS Catal., 2011, 1, 355-364.

20 M. Stalpaert, K. Janssens, C. Marquez, M. Henrion, A. L. Bugaev, A. V. Soldatov and D. De Vos, ACS Catal., 2020, 10, 9401-9409.

21 M. Stalpaert, F. G. Cirujano and D. E. De Vos, ACS Catal. 2017, 7, 5802-5809. A Inorganic, Phys. Theor., 1969, 792. 